Check for updates

Cite this: RSC Adv., 2017, 7, 23287

\title{
Resistive switching and related magnetization switching in $\mathrm{Pt} / \mathrm{BiFeO}_{3} / \mathrm{Nb}: \mathrm{SrTiO}_{3}$ heterostructures
}

\author{
Meng Zhao, ${ }^{\text {ab }}$ Yongdan Zhu, (D) $\dagger^{* a b}$ Yuan Zhang, ${ }^{a}$ Tingting Zhang, ${ }^{a}$ Da Qiu, ${ }^{a}$ \\ Guohong Lai, ${ }^{a}$ Cheng Hu, ${ }^{a}$ Qiangwen Wang, ${ }^{b}$ Feng Zhang ${ }^{b}$ and Meiya Li $\mathbb{D}^{*}$ *b
}

$\mathrm{BiFeO}_{3}$ (BFO) thin films were epitaxially grown on a $0.7 \mathrm{wt} \% \mathrm{Nb}$-doped (001) $\mathrm{SrTiO}_{3}$ (NSTO) single-crystal substrate by pulsed laser deposition to form a Pt/BFO/NSTO heterostructure. This heterostructure exhibits stable bipolar resistive switching behavior with a maximum $R_{\text {off }} / R_{\text {on }}$ ratio of $10^{5}$, well retention and multilevel memory properties. Meanwhile, the saturation magnetization $\left(M_{s}\right)$ of BFO film shows reversible switching upon different resistance states. The BFO film shows high saturation magnetization at a high resistance state, while it shows low saturation magnetization at a low resistance state. These resistive and magnetization switching properties are attributed to the modulation effect of the ferroelectric polarization reversal on the width of depletion region and the height of potential barrier via the charge trapping and detrapping process combined with the migration of oxygen vacancies at the BFO/NSTO interface. This study demonstrates that Pt/BFO/NSTO heterostructure has potential application in nonvolatile resistive switching memory and novel magnetoelectric coupling devices.

Received 11th January 2017 Accepted 20th April 2017

DOI: $10.1039 / c 7 r a 00242 d$

rsc.li/rsc-advances switching is one of the important issues to be studied at present.

BFO has been widely investigated as a promising multiferroic material because of the coexistence of ferroelectricity and magnetism, which shows potential applications in multifunctional devices. ${ }^{16-18}$ The research of BFO has been carried out in our previous studies, in which the BFO films with good ferroelectricity have been prepared successfully through pulsed laser deposition method..$^{12,19-21}$ Recently, BFO is also found to exhibit various RS behaviors due to different device structure and preparation process, which has attracted significant attention. The unipolar, bipolar and nonpolar RS behaviors have been found in the epitaxial and polycrystalline thin films even in the nano-islands and nanocapacitor arrays device structures of BFO, whose mechanisms have been mostly attributed to conductive filament, oxygen vacancy migration, charge trapping/detrapping process. ${ }^{12,14,22-26}$ However, very few works focused on the correlation and regulation behaviors between RS and magnetism switching in BFO. Recently, Sun et al., reported magnetic-field controlled RS behaviors in $\mathrm{Ag} /$ $\mathrm{BiFeO}_{3} / \gamma-\mathrm{Fe}_{2} \mathrm{O}_{3} / \mathrm{FTO}$ device, in which the extra opposite direction electrical field play a vital role in controlling the RS with the magnetic field. ${ }^{24}$ Li et al., observed resistance and magnetization switching induced by electric field in epitaxial $\mathrm{Mn}: \mathrm{ZnO} /$ $\mathrm{BiFeO}_{3}$ multiferroic heterostructures at room temperature. They suggested that the main RS and magnetic variation mechanisms were mainly ascribed to the variation of oxygen vacancies regulated by ferroelectric polarization at the $\mathrm{Mn}: \mathrm{ZnO} / \mathrm{BFO}$ interface. ${ }^{25}$ Chen $e t$ al. reported the magnetization switching in the $\mathrm{Au} / \mathrm{BiFe}_{0.9} \mathrm{Mn}_{0.1} \mathrm{O}_{3} / \mathrm{Pt}$ thin film device modulated by

\footnotetext{
${ }^{a}$ School of Information Engineer, Hubei University for Nationalities, Enshi, 44500o, Hubei, P. R. China

${ }^{b}$ School of Physics and Technology, Key Laboratory of Artificial Micro/Nano Structures of the Ministry of Education, Wuhan University, Wuhan 430072, P. R. China. E-mail: yongdan_zhu@whu.edu.cn; myli@whu.edu.cn

$\dagger$ These authors contribute equally to this work.
}

in the $\mathrm{Au} / \mathrm{BiFe}_{0.9} \mathrm{Mn}_{0.1} \mathrm{O}_{3} / \mathrm{Pt}$ thin film device modulated by 
unipolar RS process and reveal that such a magnetization switching is dominated by the conversion of Fe ion valence state between $\mathrm{Fe}^{2+}$ and $\mathrm{Fe}^{3+}$, which is accompanied with the formation and the rupture of the conduction filaments during the RS process. ${ }^{13}$ However, our and related studies have demonstrated that the $\mathrm{BFO} / \mathrm{NSTO}$ heterostructure exhibits bipolar RS effect and the ferroelectric polarization reversal on the interface play a dominant role on the RS behavior, which has a completely different mechanism from the conductive filament model., ${ }^{\mathbf{4 2 , 1 4}}$ Furthermore, study on the modulation of RS and magnetism of pure BFO films has not been reported. Therefore, in this paper, we have demonstrated the resistive and magnetism switching and the correlation between them in the Pt/BFO/NSTO heterostructure. The RS effect and the associated magnetisms as well as its modulation characteristics were investigated. The physical mechanisms of the RS and the associated magnetism modulation were explored.

\section{Experimental section}

The BFO films were epitaxially prepared on $0.7 \% \mathrm{Nb}$-doped $\mathrm{SrTiO}_{3}(100)$ single crystal substrates by pulsed laser deposition using a KrF excimer laser (Lambda Physik Compex 205) with a wavelength of $248 \mathrm{~nm}$ and a pulse frequency of $4 \mathrm{~Hz}$. During deposition, the substrate temperature, oxygen partial pressure, and deposition time were maintained at $670{ }^{\circ} \mathrm{C}, 15 \mathrm{~Pa}$, and $45 \mathrm{~min}$, respectively. After deposition, the films were cooled to room temperature at $4{ }^{\circ} \mathrm{C} \mathrm{min}^{-1}$ under an oxygen pressure of 1 atm. Pt top electrodes were deposited by DC magnetron sputtering through a multi-hole shadow mask with a $0.2 \mathrm{~mm}$ hole diameter, while the In bottom electrode was pressed on the bottom of the NSTO substrate to form an ohmic contact. The crystallographic orientation and morphology of these films were analyzed by X-ray diffraction (XRD, D8-Advance, Bruker, Germany) and scanning electron microscopy (SEM, S4800, Hitachi, Japan). Additionally, the film compositions and the chemical states of the elements were analyzed by X-ray photoelectron spectroscopy (XPS). The RS characteristics were obtained by Keithley 4200 semiconductor characterization system. The magnetic properties were measured by physical property measurement system (PPMS). All these measurements were carried out at room temperature.

\section{Results and discussion}

Fig. 1(a) shows the XRD $\theta-2 \theta$ scan of the BFO/NSTO heterostructure, the inset is the $\phi$-scans of the BFO (011) and NSTO (011) planes, respectively. The figure shows the (001) and (002) diffraction peak of BFO film and no impurity phase was observed, indicating the $c$-axis orientation of the film. A crosssectional SEM image of the heterostructure shown in Fig. 1(b) gives the BFO film a thickness of about $150 \mathrm{~nm}$. In order to determine the surface oxidation states of the BFO film, XPS measurement was performed and the results are shown in Fig. 1(c) and (d). The Fe2 $\mathrm{p}_{3 / 2}$ and O1s core-level XPS spectrum were analyzed through the Gaussian curve fitting method, and the background signal has been deducted using the Shirley method. Fig. 1(c) shows the Fe2 $\mathrm{p}_{3 / 2}$ core-level XPS spectrum of the BFO film at initial state, which is observed around $710.0 \mathrm{eV}$. And the $\mathrm{Fe} 2 \mathrm{p}_{3 / 2}$ broadband can be divided into two subbands centered at $709.2 \mathrm{eV}$ for the $\mathrm{Fe}^{2+}$ and $710.8 \mathrm{eV}$ for the $\mathrm{Fe}^{3+}$, respectively. These chemical bonding features of the $\mathrm{Fe} 2 \mathrm{p}_{3 / 2}$ are in accordance with what reported by Chen et al. ${ }^{\mathbf{1 3}}$ As shown in Fig. 1(d), the O1s core-level XPS spectra of the BFO film can be divided into two peaks located at $529.1 \mathrm{eV}$ and $531.3 \mathrm{eV}$, respectively, which usually referred to as the lattice oxygen peak and nonlattice oxygen. The results above indicate that a certain number of oxygen vacancies exist in these BFO film, which might be inevitably introduced in the preparation process.

Fig. 2(a) shows typical current-voltage $(I-V)$ characteristics of the Pt/BFO/NSTO heterostructure on a semi-log scale, the inset shows a schematic illustration of the device. A positive direction of the current was defined as from the top electrode towards the bottom electrode. The voltages were applied by the sequence of $0 \mathrm{~V} \rightarrow+3 \mathrm{~V} \rightarrow 0 \mathrm{~V} \rightarrow-5 \mathrm{~V} \rightarrow 0 \mathrm{~V}$, where the arrows indicate the sweeping direction. The device exhibits typical bipolar RS behavior without a forming process, which is opposite to the results of Chen et al. when using $\mathrm{Au} / \mathrm{BiFeMnO}_{3} / \mathrm{Pt}$ structure, indicating a different RS mechanism from the conductive filament proposed by Chen. ${ }^{13}$ As shown in Fig. 2(b), the resistances at HRS-1, HRS-2 and LRS remain unchanged after continuously reading at $-0.1 \mathrm{~V}$ for $10^{3} \mathrm{~s}$, revealing good retention characteristics, which is comparable with that obtained in the $\mathrm{Pt} / \mathrm{BFO} /$ NSTO heterostructures reported by $\mathrm{Hu}$ et al. ${ }^{\mathbf{1 2}}$ and Wang et al. ${ }^{\mathbf{1 4}}$ Furthermore, our Pt/BFO/NSTO heterostructure shows multilevel RS behavior by applied different reset voltages $\left(V_{\text {reset }}\right)$. As illustrated in Fig. 2(c), the applied sweeping voltage was applied as $+3 \mathrm{~V} \rightarrow 0 \mathrm{~V} \rightarrow V_{\text {reset }} \rightarrow 0 \mathrm{~V} \rightarrow+3 \mathrm{~V}$. When the $V_{\text {reset }}$ is $-1 \mathrm{~V}$, no obvious RS window in the $I-V$ curve is observed. However, with the increase in the $V_{\text {reset }}$, the RS window increases and the device exhibit multilevel RS effect. It is also found that in the negative region of the $I-V$ loop, an obvious negative differential resistance phenomenon is demonstrated when the $V_{\text {reset }}$ exceeds $-2 \mathrm{~V}$. The differential resistance phenomenon has also been reported in other articles, which can be explained by the variance of space charge region and trapping/detrapping of electrons by interface state..$^{\mathbf{6 , 8 , 2 7}}$

As mentioned above, BFO is a typical antiferromagnetic material in bulk form and weak ferromagnetic material in the film form. ${ }^{28}$ Therefore, it is essential to investigate the effects of RS on the magnetic properties of the BFO film. To obtain strong magnetic signals induced by the RS effect, a Pt/BFO/NSTO heterostructure with 14 resistance cells are switched consistently for the magnetization measurements. A maximum magnetic field of $1 \mathrm{~T}$ in the direction perpendicular to the film surface is applied during the measurements. The diamagnetic signals of the substrate are deducted from the raw hysteresis loops and all the measurements were carried out at room temperature. Fig. 2(d) shows the magnetization loops of the BFO film at HRS and LRS. We can see that the saturation magnetization $\left(M_{\mathrm{s}}\right)$ of the BFO film shows reversible switching upon different resistance states, giving low $M_{\mathrm{s}}$ of $45 \mu \mathrm{emu}$ at LRS while high $M_{\mathrm{s}}$ of $60 \mu \mathrm{emu}$ at HRS. The rate of change in the $M_{\mathrm{s}}$ value at LRS and HRS can reach up to $33 \%$, which is essential 

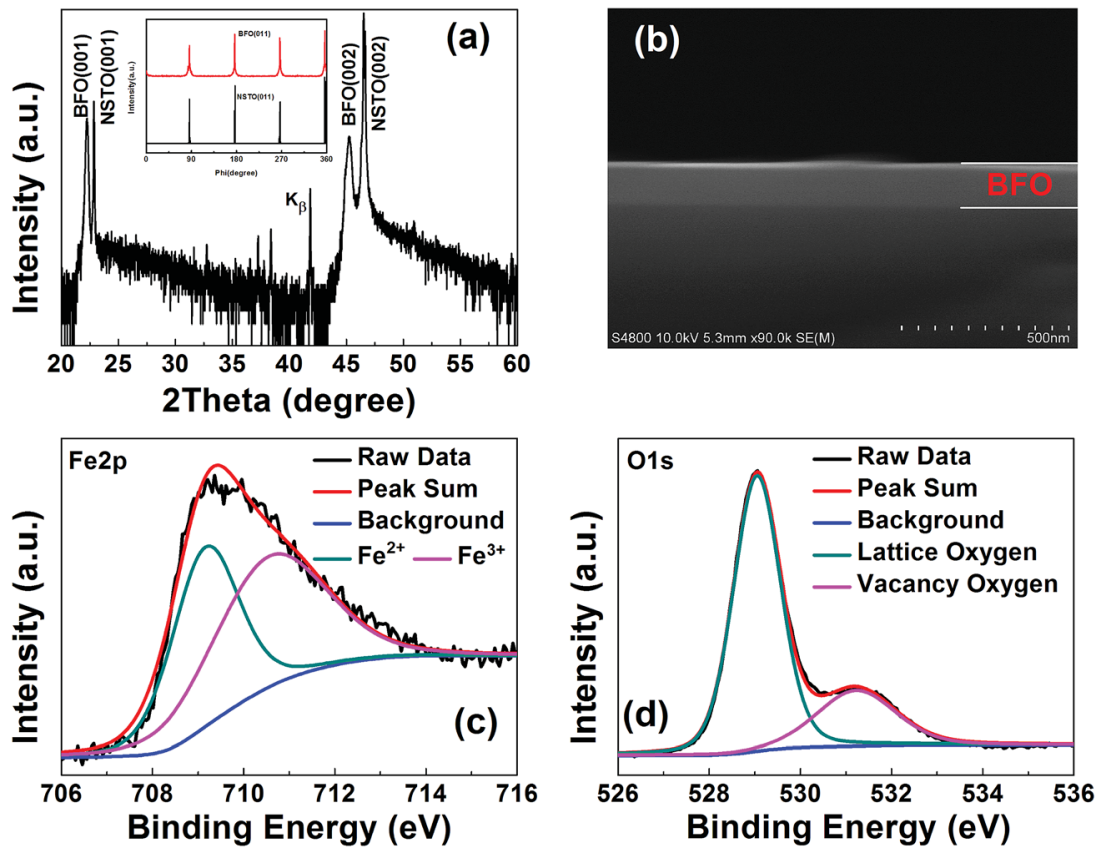

Fig. 1 (a) XRD $\theta-2 \theta$ scan of the BFO/NSTO heterostructure, the inset is the $\phi$-scans of the BFO (011) and NSTO (011) planes, respectively. (b) The cross-sectional SEM image of the heterostructure. Core-level XPS spectrum of Fe2 $\mathrm{p}_{3 / 2}$ (c) and O1s (d) of the BFO film. Each spectrum is fitted with the Gaussian curve fitting method.
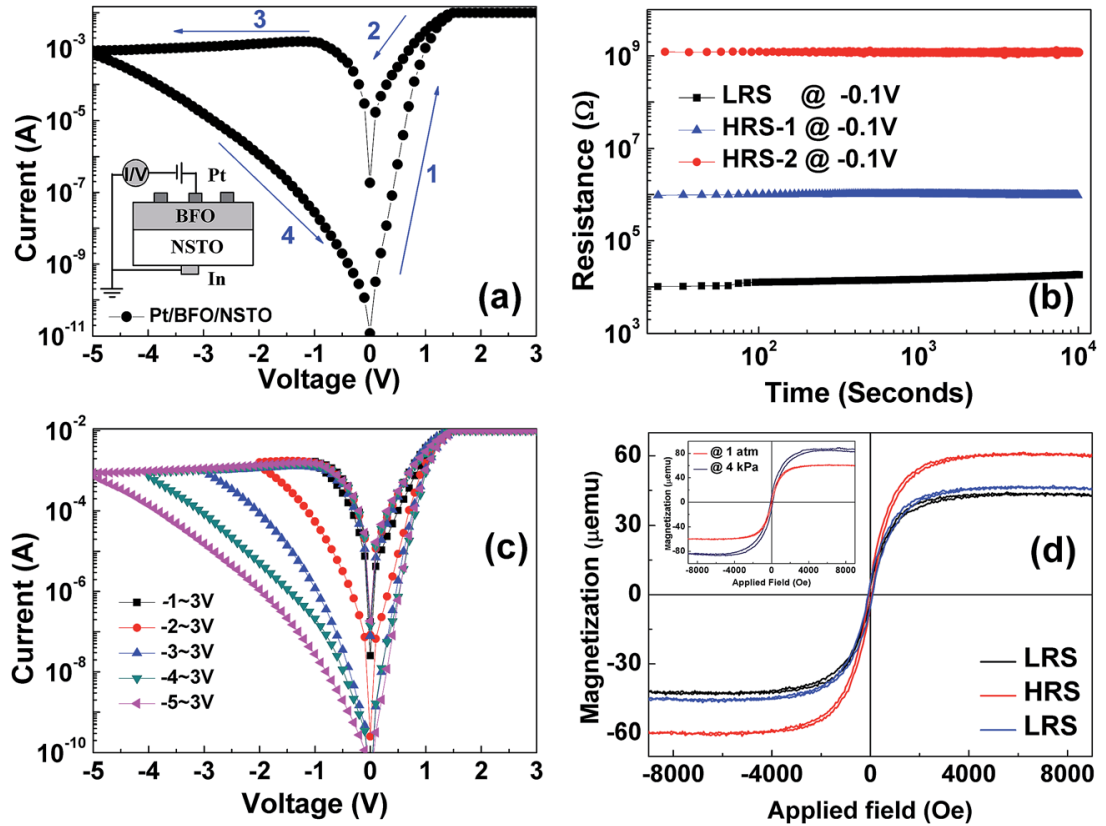

Fig. 2 (a) Typical I-V characteristics of the Pt/BFO/NSTO heterostructure in a semi-log scale, the inset shows a schematic illustration of the device. (b) Retention characteristics of the device at HRS-1, HRS-2 and LRS. (c) Multilevel RS behavior of the device. (d) Magnetic loops of the device at HRS and LRS. The inset is magnetic loops of BFO film that cooling in different oxygen pressure of 1 atm and $4 \mathrm{kPa}$.

for practical applications. The coexistence of resistive and magnetization switching in the BFO film provides a possible way to design switchable multilevel memory and multi-field signal detection devices via both electricity and magnetism.

Previous studies have shown that the variation of magnetism in the oxide heterostructure is closely relevant to the content of oxygen vacancies in the oxide film. ${ }^{6,8,11}$ In order to investigate the role of the oxygen vacancies in regulation of the magnetism of the Pt/BFO/NSTO structure, we prepared another BFO sample under the same deposition conditions which was cooled in lower oxygen pressure of $4 \mathrm{kPa}$. As shown in the inset of Fig. 2(d), the magnetization of the sample in a higher cooling 
oxygen pressure of 1 atm is obviously smaller than that in a lower cooling oxygen pressure of $4 \mathrm{kPa}$. As the previous study has shown that the cooling process in a lower oxygen pressure can introduce more oxygen vacancies in the interior of the BFO films than that in a higher oxygen pressure. ${ }^{29}$ This might imply that the concentration of oxygen vacancies in the BFO films show a close relationship with the corresponding magnetism.

In order to further investigate the physical mechanism related to resistive and magnetization switching of the $\mathrm{Pt} / \mathrm{BFO} /$ NSTO heterostructure, analysis on the internal conductive transport properties is necessary. The most possible conductive mechanisms include space-charge-limited conduction (SCLC), Schottky emission (SE) and Poole-Frenkel emission (PF). ${ }^{30-32}$ Fig. 3(a) plots the $I-V$ curves of the Pt/BFO/NSTO heterostructure at positive bias region. It shows that the $I-V$ transport characteristics belongs to the SCLC, which is consistent with other research reports. ${ }^{27}$ This suggests that the defects in the films such as oxygen vacancies play an important role in the transport process. Fig. 3(b) shows the $\ln (-I)$ vs. $\ln (-V)$ plot based on the data from Fig. $2(\mathrm{a})(0 \mathrm{~V}$ to $-1 \mathrm{~V})$ in the negative bias region of the LRS. The curve shows linear behavior with a slope of 2 , corresponding to the SCLC mechanism. Fig. 3(c) and (d) show the $\ln (I / V)$ versus $V^{1 / 2}$ plots based on the data from Fig. 2(a) $(-1$ to $-5 \mathrm{~V}$ and $-5 \mathrm{~V}$ to $0 \mathrm{~V}$, respectively). The curves show linear behavior, corresponding to the PF emission mechanism. Therefore, the conduction behavior of the set process and the LRS can be explained using SCLC mechanism, and the HRS in the negative voltage region can be explained by PF emission. This behavior is also consistent with that reported previously on interfacial effects, where oxygen vacancy migration and charge trapping/detrapping at the interface drive RS in various heterostructures. ${ }^{\mathbf{6 , 8 , 1 1 , 3 0}}$
Based on the experimental results and analysis above, the correlation changes of the resistance and magnetism of the devices can be attributed to the modulation effect of the ferroelectric polarization reversal on the width of depletion region and the height of potential barrier via the charge trapping and detrapping process combined with the migration of oxygen vacancies at the BFO/NSTO interface. The Fig. 4 shows the schematic diagrams of the energy band of RS and magnetization switching of the Pt/BFO/NSTO device. As shown in Fig. 4(a), when the positive voltage is applied to the device, the ferroelectric polarization in the BFO film points downward. Because of the ferroelectric field effect, the oxygen vacancies migrate towards the interface of the BFO/NSTO and the electrons in NSTO would inject into the depletion layer at the interface, resulting in the decreases in the depletion width and the barrier height. Subsequently, the electrons would pass over the barrier and inject into the BFO films, and eventually, be trapped by the oxygen vacancies, which correspond to the RS behavior from HRS to LRS. As can be seen in Fig. 4(b), when the reverse voltage was applied to the device, the ferroelectric polarization in the BFO films points upward, resulting in the increase in the depletion width and the height of the barrier. At the same time, the electrons might be detrapped and migrate towards the bottom electrode while the oxygen vacancies migrate backwards into the BFO films, which correspond to the RS from LRS to HRS.

Typical BFO crystal exhibits a rhombohedral phase $(R 3 c)$ and a G-type antiferromagnetic structure with spiral spin modulation cycle of $62 \mathrm{~nm}$, which usually can not show strong ferromagnetism. ${ }^{28}$ However, in the previous studies, the observed ferromagnetic behaviors of BFO thin films was attributed to the effect of the mismatch strain or the change of Fe ion valence
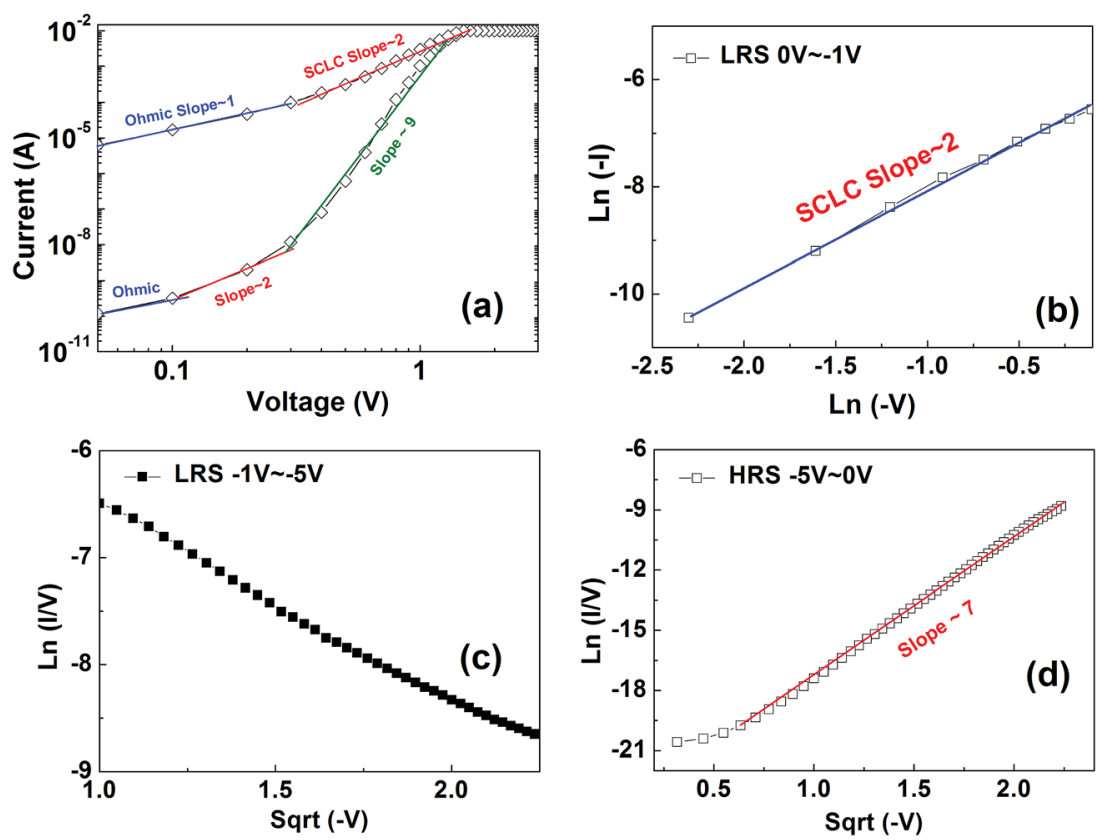

Fig. 3 Plots of the I-V curves in Fig. 2(a) on different scales to reveal their conduction mechanisms: (a) positive bias region plotted in log-log scale; (b) $\ln (-l)$ vs. $\ln (-V)$ in the negative bias region $(0$ to $-1 \mathrm{~V})$ at LRS. (c) $\ln (/ / \mathrm{V}) \mathrm{vs}$. $\left(-V^{1 / 2}\right.$ in the negative bias region $(-1$ to $-5 \mathrm{~V})$. (d) $\ln (/ / \mathrm{V})$ vs. $(-V)^{1 / 2}$ in the negative bias region $(-5$ to $0 \mathrm{~V})$. 




(a) LRS

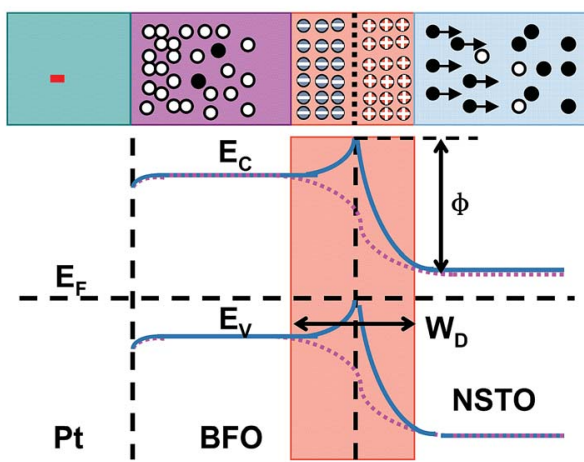

(b) HRS

Fig. 4 Schematic charge distribution and energy diagrams of the Pt/ BFO/NSTO heterostructure at LRS and HRS. The "plus" and "minus" symbols represent ionized acceptors and donors; the white circle and black dot symbols represent holes and electrons, respectively; the circle with the black dot in its center symbols represent electrons trapped by the oxygen vacancies.

state. ${ }^{33-36}$ Based on the first-principle calculations, Chen et al. found that Mn doping in the BFO films could lead to easy conversion of $\mathrm{Fe}$ ion between $\mathrm{Fe}^{3+}$ and $\mathrm{Fe}^{2+}$ because of the impurity energy level. ${ }^{13}$ Thus, the initial states of these films exhibit ferromagnetism. As for our BFO films, we speculate that the origin of ferromagnetism may be due to the change of valence state of iron ions because of inevitable oxygen vacancies introduced in the deposition process. Under the positive voltage, the device is set from HRS to LRS. Due to the ferroelectric field effect, the oxygen vacancies migrate towards the bottom electrode and gathering at the interface, while the injected electrons would neutralize the positive charge of the oxygen vacancies, and thus the number of oxygen vacancies in the internal of BFO films would decrease. The reduction of oxygen vacancies results in oxidation of partial $\mathrm{Fe}^{2+}$ to $\mathrm{Fe}^{3+}$, which would weaken the double exchange interaction between $\mathrm{Fe}^{2+}$ and $\mathrm{Fe}^{3+}$. Accordingly, the $M_{\mathrm{S}}$ in the LRS decreases. However, when the device is reset from LRS to HRS under the negative voltage, the oxygen vacancies at the interface detrap electrons and migrate backwards into the BFO films, which increases the concentration of oxygen vacancies in BFO films. Thus, partial $\mathrm{Fe}^{3+}$ are reduced to $\mathrm{Fe}^{2+}$, which could enhance the double exchange interaction between $\mathrm{Fe}^{2+}$ and $\mathrm{Fe}^{3+}$. Then the $M_{\mathrm{s}}$ in the HRS increases. Therefore, we achieve the regulation of both RS and $M_{\mathrm{s}}$ by the electric field, which exhibits nonvolatile, reversible and repeatable characteristics.

\section{Conclusions}

In conclusion, our Pt/BFO/NSTO device shows stable bipolar resistive switching characteristics with maximum $R_{\mathrm{off}} / R_{\mathrm{on}}$ ratio of $10^{5}$, good retention and multilevel memory properties. Moreover, the saturation magnetization of BFO film shows reversible switching associated with its resistance states. These resistive and magnetization switching characteristics can be attributed to the modulation effect of the ferroelectric polarization reversal on the width of depletion region and the height of potential barrier via the charge trapping and detrapping process combined with the migration of oxygen vacancies at the $\mathrm{BFO} /$ NSTO interface. The results of this study extend the application field of BFO in multiferroic RS multifunctional devices.

\section{Acknowledgements}

This work was supported by the National Natural Science Foundation of China (Grant No. 11504101, 51372174, and 11364018), the Natural Science Foundation of Hubei Province (Grant No. 2014CFB610) and the Excellent Young Innovation Team Project of Hubei Province (Grant No. T201429).

\section{References}

1 G. I. Meijer, Science, 2008, 319, 1625.

2 D. B. Strukov, G. S. Snider, D. R. Stewart and R. S. Williams, Nature, 2008, 453, 80.

3 A. Sawa, Mater. Today, 2008, 11, 28.

4 T. L. Qu, Y. G. Zhao, D. Xie, J. P. Shi, Q. P. Chen and T. L. Ren, Appl. Phys. Lett., 2011, 98, 173507.

5 L. Wang, K. Jin, C. Ge, C. Wang, H. Guo, H. Lu and G. Yang, Appl. Phys. Lett., 2013, 102, 252907.

6 S. Ren, H. Qin, J. Bu, G. Zhu, J. Xie and J. Hu, Appl. Phys. Lett., 2015, 107, 062404.

7 S. Ren, L. Zhang, J. Dong, Y. Huang, J. Guo, L. Zhang, J. Zhao, X. Zhao and W. Chen, J. Mater. Chem. C, 2015, 3, 4077.

8 M. Zhao, Y. Zhu, Q. Wang, M. Wei, X. Liu, F. Zhang, C. Hu, T. Zhang, D. Qiu, M. Li and R. Xiong, Appl. Phys. Lett., 2016, 109, 013504.

9 Y. Q. Xiong, W. P. Zhou, Q. Li, M. C. He, J. Du, Q. Q. Cao, D. H. Wang and Y. W. Du, Appl. Phys. Lett., 2014, 105, 032410.

10 Y. Luo, D. Zhao, Y. Zhao, F. Chiang, P. Chen, M. Guo, N. Luo, X. Jiang, P. Miao, Y. Sun, A. Chen, Z. Lin, J. Li, W. Duan, J. Cai and Y. Wang, Nanoscale, 2015, 7, 642.

11 Q. Wang, Y. Zhu, X. Liu, M. Zhao, M. Wei, F. Zhang, Y. Zhang, B. Sun and M. Li, Appl. Phys. Lett., 2015, 107, 063502.

12 Z. Q. Hu, Q. Li, M. Y. Li, Q. W. Wang, Y. D. Zhu, X. L. Liu, X. Z. Zhao, Y. Liu and S. X. Dong, Appl. Phys. Lett., 2013, 102, 102901.

13 G. Chen, G. Bi, L. Song, Y. Weng, D. Pan, Y. Li, S. Dong, T. Tang, J. M. Liu and J. G. Wan, Appl. Phys. Lett., 2016, 109, 112903. 
14 C. Wang, K. Jin, Z. Xu, L. Wang, C. Ge, H. Lu, H. Guo, M. He and G. Yang, Appl. Phys. Lett., 2011, 98, 192901.

15 Y. Huang, Y. Wu, X. Wang, S. Zhang, X. Shia and H. Zeng, RSC Adv., 2016, 6, 17867.

16 T. Zhao, A. Scholl, F. Zavaliche, K. Lee, M. Barry, A. Doran, M. Cruz, Y. Chu, C. Ederer and N. Spaldin, Nat. Mater., 2006, 5, 823.

17 R. Ramesh and N. A. Spaldin, Nat. Mater., 2007, 6, 21.

18 Y. H. Chu, L. W. Martin, M. B. Holcomb, M. Gajek, S. J. Han, Q. He, N. Balke, C. H. Yang, D. Lee and W. Hu, Nat. Mater., 2008, 7, 478.

19 Z. Hu, M. Li, Y. Zhu, S. Pu, X. Liu, B. Sebo, X. Zhao and S. Dong, Appl. Phys. Lett., 2012, 100, 252908.

20 B. F. Yu, M. Y. Li, J. Wang, Z. Q. Hu, X. L. Liu, Y. D. Zhu and X. Z. Zhao, Thin Solid Films, 2012, 520, 4089.

21 B. Yu, M. Li, Z. Hu, L. Pei, D. Guo, X. Zhao and S. Dong, Appl. Phys. Lett., 2008, 93, 182909.

22 J. Luo, S. Lin, Y. Zheng and B. Wang, Appl. Phys. Lett., 2012, 101, 062902.

23 J. H. Jeon, H. Y. Joo, Y. M. Kim, D. H. Lee, J. S. Kim, Y. S. Kim, T. Choi and B. H. Park, Sci. Rep., 2016, 6, 23299.

24 B. Sun, Y. Liu, W. Zhao and P. Chen, $R S C A d v .$, 2015, 5, 13513.

25 D. Li, W. Zheng, D. Zheng, J. Gong, L. Wang, C. Jin, P. Li and H. Bai, ACS Appl. Mater. Interfaces, 2016, 8, 3977.
26 L. Zhao, Z. Lu, F. Zhang, G. Tian, X. Song, Z. Li, K. Huang, Z. Zhang, M. Qin, S. Wu, X. Lu, M. Zeng, X. Gao, J. Dai and J. M. Liu, Sci. Rep., 2015, 5, 09680.

27 P. Wang, P. Li, Y. Zhi, D. Guo, A. Pan, J. Zhan, H. Liu, J. Shen and W. Tang, Appl. Phys. Lett., 2015, 107, 262110.

28 Y. H. Chu, L. W. Martin, M. B. Holcomb and R. Ramesh, Mater. Today, 2007, 10, 16.

29 G. L. Yuan, L. W. Martin, R. Ramesh and A. Uedono, Appl. Phys. Lett., 2009, 95, 012904.

30 H. Peng, G. Li, J. Ye, Z. Wei, Z. Zhang, D. Wang, G. Xing and T. Wu, Appl. Phys. Lett., 2010, 96, 192113.

31 T. Fujii, M. Kawasaki, A. Sawa, Y. Kawazoe, H. Akoh and Y. Tokura, Phys. Rev. B: Condens. Matter Mater. Phys., 2007, 75, 165101.

32 A. Odagawa, H. Sato, I. H. Inoue, H. Akoh, M. Kawasaki, Y. Tokura, T. Kanno and H. Adachi, Phys. Rev. B: Condens. Matter Mater. Phys., 2004, 70, 224403.

33 J. Wang, J. B. Neaton, H. Zheng, V. Nagarajan, S. B. Ogale, B. Liu, D. Viehland, V. Vaithyanathan, D. G. Schlom, U. V. Waghmare, N. A. Spaldin, K. M. Rabe, M. Wuttig and R. Ramesh, Science, 2003, 299, 1719.

$34 \mathrm{~W}$. Eerenstein, F. D. Morrison, J. Dho, M. G. Blamire, J. F. Scott and N. D. Mathur, Science, 2005, 307, 1203.

35 C. Ederer and N. A. Spaldin, Phys. Rev. B: Condens. Matter Mater. Phys., 2005, 71, 224103.

36 S. K. Kim, H. M. Jang and J. Chang, Journal of Magnetics, 2006, 11, 108. 